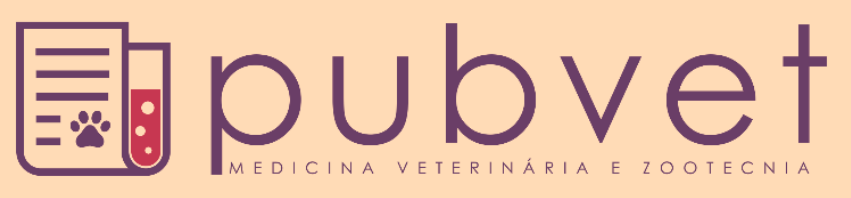

https://doi.org/10.31533/pubvet.v15n07a870.1-6

\title{
Persistência do ducto arterioso em cão: Relato de caso
}

\author{
Luana Reis Teixeira Macêdo ${ }^{1 *} \bullet$, Rômulo Ferreira de Assumção ${ }^{2} \theta$, Rodrigo Pereira Brum ${ }^{3 \bullet}$, Fabiana \\ Gaspar da Cunha ${ }^{4}$, Marcos Augusto Lopes de Castro ${ }^{50}$, Liliane Maria Valentim Willi Monteiro ${ }^{60}$
}

\author{
${ }^{1}$ Graduanda em Medicina Veterinária na Universidade Grande Rio - UNIGRANRIO, Duque de Caxias - RJ, Brasil. \\ ${ }^{2}$ Professor mestre do curso de medicina veterinária da Universidade Iguaçu - UNIG, Nova Iguaçu - RJ, Brasil. \\ ${ }^{3}$ Médico Veterinário graduado pela Universidade Estácio de Sá - UNESA, Vargem Pequena - RJ, Brasil. \\ ${ }^{4}$ Médica veterinária graduada pela Universidade Castelo Branco - UCB, Penha- RJ, Brasil. \\ ${ }^{5}$ Médico Veterinário graduado pela Universidade Federal Fluminense - UFF, Niteroi - RJ. \\ ${ }^{6}$ Professora doutora do curso de medicina veterinária da Universidade do Grande Rio - UNIGRANRIO, Duque de Caxias - RJ, Brasil. \\ *Autor para correspondência, E-mail: luanareisvet@gmail.com
}

Resumo. O objetivo deste trabalho foi relatar um caso de persistência do ducto arterioso (PDA) em cão, visto a importância do diagnóstico precoce e posterior correção cirúrgica para aumento de perspectiva de vida do animal. Essa anomalia cardíaca é caracterizada pelo não fechamento do ducto arterioso que deve ocorrer de forma fisiológica após nascimento do feto. Um canino macho da raça Spitz Alemão, com cinco meses de idade, não castrado, foi submetido ao atendimento clínico para consulta pediátrica. Durante o exame físico foi detectado sopro à auscultação cardíaca. Diante deste achado foi indicado para avaliação cardiológica. Para isto, foi realizado ecodopplercardiograma, que possibilitou o diagnóstico de persistência de ducto arterioso. Após o diagnóstico foi sugerido tratamento cirúrgico, no qual foi realizada quinze dias após o exame de imagem. O procedimento cirúrgico foi realizado por meio de toracotomia lateral esquerda para acesso à artéria aorta e artéria pulmonar, possibilitando a oclusão do ducto, feita com fio seda 2-0. No pós-operatório imediato, o paciente foi encaminhado para internação, sendo liberado para seu domicílio após 24 horas. Durante avaliação clínica e novo exame ecodopplercardiograma não foi evidenciado a presença do PDA e não foi auscultado sopro cardíaco caracterizando êxito cirúrgico. Desta forma, foi possível concluir que a cirurgia para correção do ducto foi fundamental para possibilitar o aumento na expectativa $\mathrm{e}$ qualidade de vida do animal.

Palavras chave: Anomalia cardíaca congênita, eccodopplercardiograma, PDA

\section{Persistent ductus arteriosus in a dog: Case report}

\begin{abstract}
The aim of this study was to report a case of persistent ductus arteriosus (PDA) in a dog, given the importance of early diagnosis and subsequent surgical correction to increase the animal's outlook on life. This cardiac anomaly is characterized by the nonclosure of the ductus arteriosus, which must occur physiologically after the birth of the fetus. A five-month-old male German Spitz canine, not castrated, underwent clinical care for pediatric consultation. During the physical examination, a murmur to cardiac auscultation was detected. In view of this finding, he was indicated for cardiological evaluation. For this, an echocardiogram was performed, which enabled the diagnosis of persistent ductus arteriosus. After the diagnosis, surgical treatment was suggested, which was performed fifteen days after the imaging exam. The surgical procedure was performed by means of left lateral thoracotomy to access the aorta and pulmonary arteries, allowing duct occlusion, made with 2-0 silk thread. In the immediate postoperative period, the patient was referred for admission, being released to his home after 24 hours. During clinical evaluation and a new Doppler echocardiogram, the presence of the PDA was not
\end{abstract}


evidenced and a heart murmur was not heard, characterizing surgical success. Thus, it was possible to conclude that surgery to correct the duct was fundamental to enable an increase in the expectation and quality of life of the animal.

Keywords: Congenital cardiac anomaly, echodopplercardiogram, PDA

\section{Conducto arterioso persistente en un perro: Reporte de un caso}

Resumen. El objetivo de este estudio fue reportar un caso de conducto arterioso persistente (DAP) en un perro, dada la importancia del diagnóstico precoz y posterior corrección quirúrgica para incrementar la perspectiva de vida del animal. Esta anomalía cardíaca se caracteriza por el no cierre del conducto arterioso, que debe ocurrir fisiológicamente después del nacimiento del feto. Un canino Spitz alemán macho de cinco meses, no castrado, fue sometido a cuidados clínicos para consulta pediátrica. Durante la exploración física se detectó soplo a la auscultación cardíaca. Ante este hallazgo, fue indicado para evaluación cardiológica. Para ello se llevó a cabo un ecocardiograma que permitió el diagnóstico de persistencia del conducto arterioso. Tras el diagnóstico se sugirió tratamiento quirúrgico, que se realizó quince días después del examen de imagen. El procedimiento quirúrgico se realizó mediante toracotomía lateral izquierda para acceder a la aorta y arterias pulmonares, permitiendo la oclusión del conducto, realizada con hilo de seda 2-0. En el postoperatorio inmediato, el paciente se remitió para su ingreso, siendo dado de alta a su domicilio a las 24 horas. Durante la evaluación clínica y un nuevo ecocardiograma Doppler, no se evidenció la presencia del CAP y no se escuchó el soplo cardíaco, caracterizando el éxito quirúrgico. Así, se pudo concluir que la cirugía para corregir el conducto es fundamental para permitir un aumento en la expectativa y calidad de vida del animal.

Palabras clave: Anomalía cardíaca congénita, ecodopplercardiograma, CAP

\section{Introdução}

A persistência do ducto arterioso (PDA) é uma anomalia congênita comum em cães (Orton, 1997; Saunders et al., 1999). Trata-se de uma estrutura presente durante a vida fetal de mamíferos, responsável por ligar a artéria pulmonar à artéria aorta, permitindo assim que o sangue contorne o pulmão (Dehkordi \& Hoseini, 2016; Ecco et al., 2008; Gournay, 2011; Lee \& Kim, 2004). De acordo com Belerenian et al. (2003) e (Buchanan \& Patterson, 2003) o sangue fetal passa da artéria pulmonar para a aorta através do ducto arterioso já que a pressão pulmonar se encontra maior que a pressão sistêmica. Após o nascimento os pulmões se enchem de ar e nesse momento a pressão pulmonar diminui permitindo a entrada de fluxo sanguíneo nos pulmões e a pressão sistêmica aumenta, estabelecendo um fluxo sanguíneo compatível com a vida.

O ducto se fecha algumas horas após o nascimento, quando isso não ocorre temos o chamado ducto arterioso persistente que causa alterações hemodinâmicas, resultando em shunt da artéria aorta para a artéria pulmonar. Em consequência disso, leva à sobrecarga do ventrículo esquerdo, dilatação e hipertrofia do mesmo (Fossum, 2014). Animais com essa anomalia podem apresentar intolerância ao exercício físico, tosse, taquipneia e em alguns casos esses pacientes são assintomáticos (Belerenian et al., 2003; Strickland, 2008).

De acordo com Broaddus \& Tillson (2010) a suspeita dessa anomalia se inicia na auscultação torácica determinando a importância de um exame clínico completo em animais jovens. Após detectar sopro cardíaco, o animal deve seguir para exames complementares, nos quais temos o ecodopplercardiograma como exame para o diagnóstico definitivo (Bonagura \& Lehmkukl, 1999). O tratamento é feito com intervenção cirúrgica, no qual o procedimento é realizado pela ligadura do ducto, sendo esse um método seguro e eficaz (Johnson, 2007).

Esse trabalho tem como objetivo relatar um caso de PDA em cão visto a importância da resolução clínica e cirúrgica dessa anomalia para aumento de perspectiva de vida para o animal. 


\section{Relato de caso}

Canino macho da raça Spitz Alemão, cinco meses de idade, inteiro, pesando 1,9kg, foi submetido à avaliação clínica e vacinação no dia 20/06/2020. Na anamnese foi relatado que o animal apresentava normoquezia, normofagia, normouria, normodipsia e sem queixas no comportamento. No exame físico, o animal encontrava-se normotenso, mucosas normocoradas, normohidratado, sem alterações em linfonodos e ausculta respiratória normal. Na ausculta cardíaca foi constatado sopro grau IV. Diante deste achado clínico, foi indagado ao tutor se o animal apresentava tosse, havendo confirmação deste sinal clínico e informado que ocorriam principalmente após exercício físico foi orientado a realização de um ecodopplercardiograma como exame complementar, para melhor avaliação do paciente.

Durante a avaliação cardíaca foi possível detectar insuficiência de mitral discreta, sobrecarga volumétrica de ventrículo esquerdo, padrão de enchimento de $\mathrm{AE}$ aumentado com velocidade de onda E mitral $=1,26 \mathrm{~m} / \mathrm{s}$ e persistência do Ducto Arterioso Tipo 1, com shunt esquerda direita (figura 1) e gradiente máximo de $88 \mathrm{mmHg}$, ducto com $0,2 \mathrm{~cm}$ de abertura e $0,4 \mathrm{~cm}$ de ampola com isso foi fechado diagnóstico persistência do ducto arterioso (PDA) esquerda direita. Ao término do exame, foi informado ao tutor que o animal precisaria de intervenção cirúrgica. Iniciou-se medicação oral paliativa com pimobendan $(0,25-0,3 \mathrm{mg} / \mathrm{kg} \mathrm{BID}, \mathrm{VO})$ usado como auxílio devido a insuficiência cardíaca congestiva e valvar por ser um inotrópico positivo e vasodilatador e também foi prescrito espironolactona $(0,5-$ $2,0 \mathrm{mg} / \mathrm{kg} \mathrm{SID}, \mathrm{VO}$ ) devido hipertensão, por se tratar de um fármaco diurético.

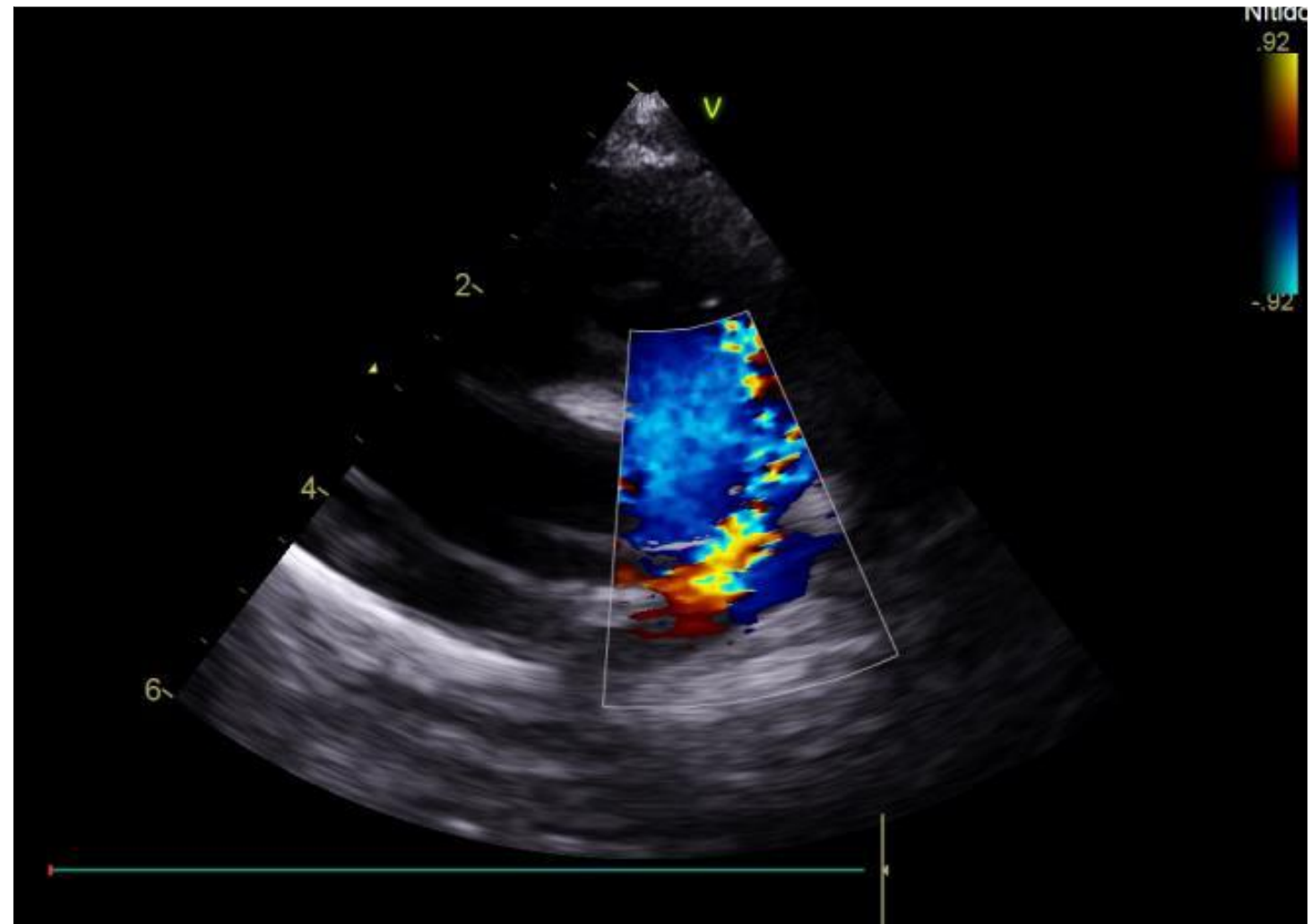

Figura 1. Imagem ecodopplercardiograma do canino do estudo, com cinco meses de idade, evidenciando shunt esquerda direita, ou seja, da aorta para a tronco pulmonar, caracterizando a persistência do ducto arterioso.

Quinze dias após o diagnóstico de PDA, o animal foi encaminhado para realização da cirurgia (figura 2). O animal foi submetido a protocolo anestésico de indução com isoflurano a $6 \%$ e oxigênio a $94 \%$ pela máscara, no trans operatório inicial foi feito tramadol $(5 \mathrm{mg} / \mathrm{kg} \mathrm{SC})$, dexametasona $(0,5 \mathrm{mg} / \mathrm{kg} \mathrm{IV})$ e dipirona ( $25 \mathrm{mg} / \mathrm{kg} \mathrm{IV})$, foi feito também bloqueio intercostal com lidocaína ( $2 \mathrm{mg} / \mathrm{kg} \mathrm{SC})$ e morfina epidural $(0,2 \mathrm{mg} / \mathrm{kg})$ em espaço sacrococcígeo e para manutenção do plano anestésico foi utilizado isoflurano a $2,5 \%$.

O procedimento cirúrgico foi realizado através de toracotomia lateral esquerda ( $4^{\circ}$ espaço intercostal), e colocação do afastador de Finochetto, rebatimento dos lobos pulmonares para visualização da base do coração onde foi evidenciado a presença de um aneurisma no tronco pulmonar, que dificultou a dissecção 
cirúrgica. Foi feito isolamento do nervo vago com fita umbilical e prosseguindo com dissecção cautelosa do ducto utilizando a pinça Mixter, possibilitando a realização da ligadura, feita com fio seda 2-0 em dois pontos (dorsal e ventral) ocluindo o vaso. Nesse momento o animal apresentou uma arritmia já esperada pela oclusão do ducto aumentando o fluxo e pressão em artéria aorta, o que necessita uma maior força dos ventrículos, animal estabilizou e de imediato foi possível verificar através da palpação da artéria aorta e artéria pulmonar que não havia mais a presença do frêmito. Em seguida foi feito colocação de dreno torácico e posteriormente rafia dos tecidos (Figura 3).

No pós-cirúrgico imediato, foi observado hiperventilação. O animal se apresentou normotenso, glicemia 137 e temperatura de $39^{\circ} \mathrm{C}$, foi ofertado oxigênio através de máscara até estabilização do quadro que ocorreu em alguns minutos. O paciente ficou em observação na internação onde continuou com as medicações orais já mencionadas, além de antibioticoterapia com ceftriaxona $(30 \mathrm{mg} / \mathrm{kg}, \mathrm{BID}$, $\mathrm{SC})$, controle da dor com tramadol (3 mg/kg, BID, IV), dipirona ( $25 \mathrm{mg} / \mathrm{kg}$, TID, IV) e dexametasona $(0,5 \mathrm{mg} / \mathrm{kg}$, SID, IV). Durante a internação animal se manteve estável, o dreno foi retirado após três horas de cirurgia e a alta do paciente ocorreu após 24 horas seguintes.

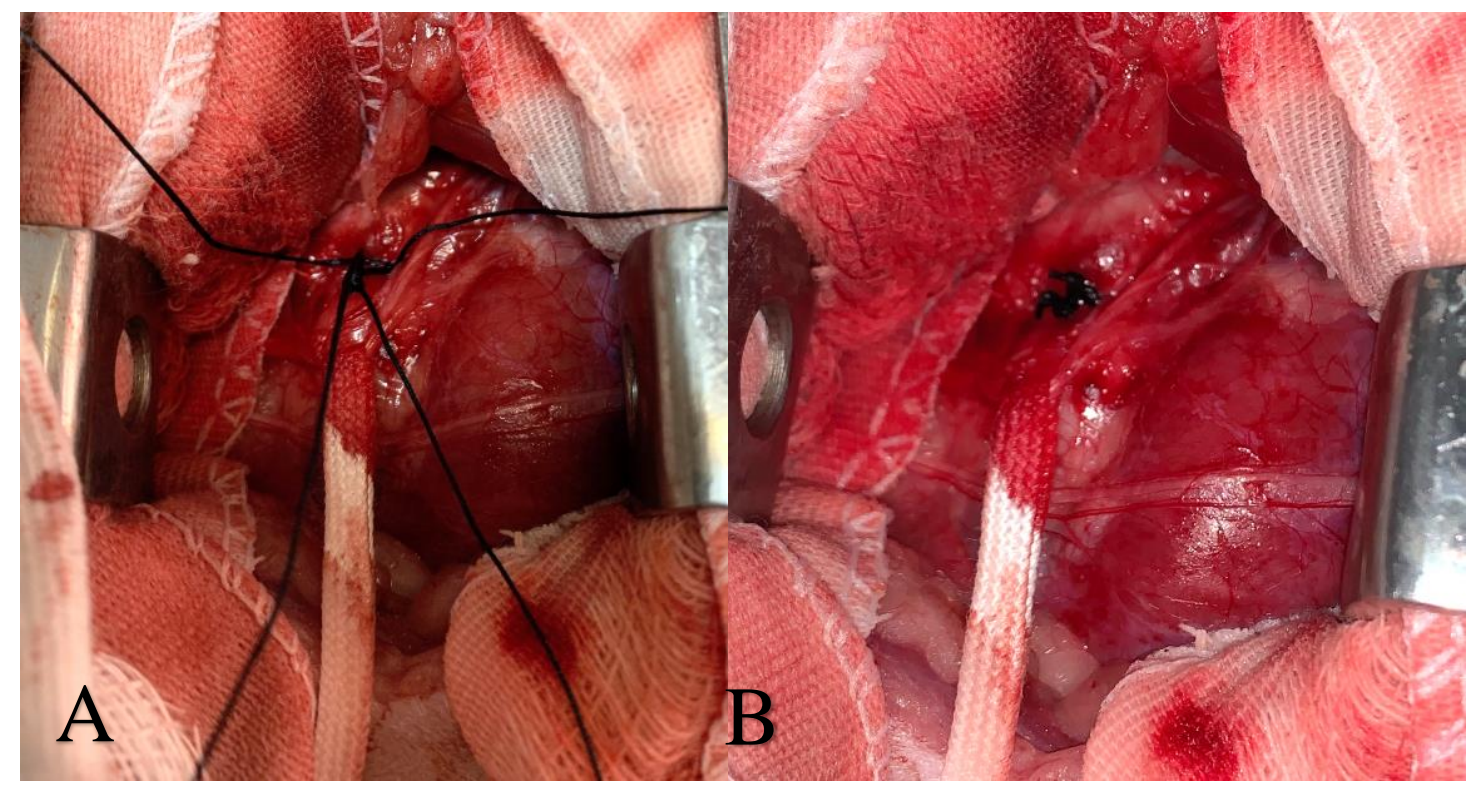

Figura 2. Imagem da ligadura do ducto arterioso durante o procedimento cirúrgico de um cão, com cinco meses de idade. Representado na imagem (A) o momento de preparo e posicionamento dos fios e na imagem (B) o momento de ligadura.

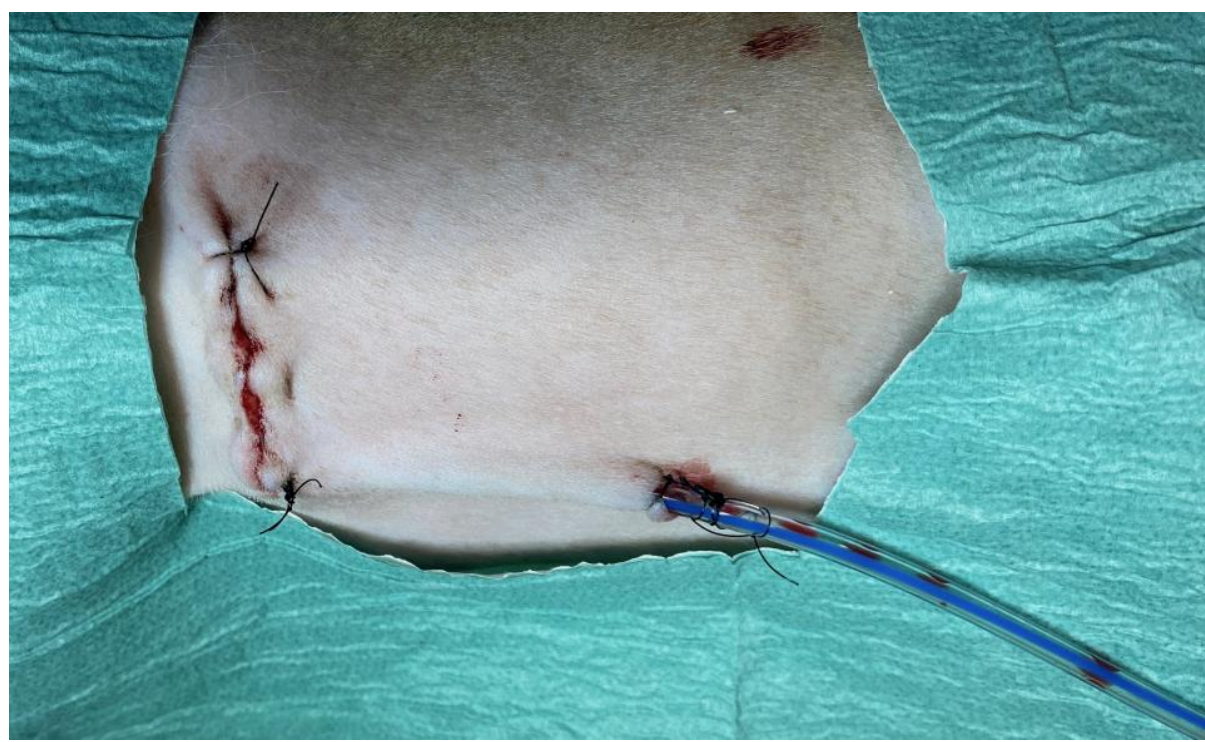

Figura 3. Imagem do dreno colocado no canino, com cinco meses de idade, após fechamento do quadrante torácico. 
Foi realizado ecodopplercardiograma 24 horas após o procedimento para avaliação pós-cirúrgica. No exame, o animal apresentou discreta sobrecarga de ventrículo esquerdo, discreto e brando refluxo em mitral, função diastólica e sistólica com discreto déficit e com gradientes de pressão dentro dos padrões e já não foi visualizado o aneurisma sugerindo que com o restabelecimento da pressão e do fluxo o mesmo regrediu. Foi recomendada reavaliação em 30 dias.

Dez dias após alta cirúrgica o animal voltou para retirada dos pontos, apresentando bom estado geral e ausência de sopro na ausculta cardíaca. Após três meses animal retornou para novo ecodopplercardiograma no dia 20/09/2020, no qual foi evidenciado aumento na dimensão do ventrículo esquerdo, com velocidade de onda $\mathrm{E}$ mitral $=0,76 \mathrm{~m} / \mathrm{s}$ e discreto aumento de volume diastólico de ventrículo esquerdo. No decorrer do exame e avaliação clínica o animal estava ativo, não foi auscultado sopro cardíaco e o mesmo não apresentava mais os quadros de tosse após exercício físico.

\section{Discussão}

A persistência do ducto arterioso (PDA), trata-se de uma cardiopatia que normalmente acomete exemplares das raças Maltês, Sptiz Alemão, Chihuahua, Pastor de Shetland, English Springer Spaniels, Bichon Frisé, Poodle, Keeshound e Yorkshire (Bonagura, 1992). Neste caso relatado, a raça em questão foi Spitiz Alemão, sendo uma das citadas pela literatura consultada.

Segundo Fossum (2014) e Snaps et al. (1995) a sintomatologia apresentada por animais acometidos com essa anomalia é intolerância ao exercício, tosse e fadiga. $\mathrm{O}$ animal do estudo apresentava quadros de tosse após exercícios físicos, o que levou a suspeita de uma cardiopatia e consequentemente a indicação e realização do exame de imagem ecodopplercardiograma.

O exame de imagem denominado ecodopplercardiograma é considerado definitivo para fechar diagnóstico por proporcionar a visualização do aumento atrial esquerdo, aumento do ventrículo esquerdo, conformidade ou não do fluxo de ejeção da aorta, definir as dimensões do tamanho do ducto e classifica-lo como um PDA clássico no qual temos o shunt esquerda direita ou um PDA reverso no qual teremos um shunt direita esquerda e um pior prognóstico para o animal (Bojrab, 2005). Nesse contexto, após a realização do exame de imagem, foi possível fechar o diagnóstico de PDA com shunt esquerda direita, ou seja, de artéria aorta para tronco pulmonar através do doppler, ventrículo esquerdo com dimensões aumentadas e sobrecarga volumétrica, refluxo discreto em mitral e ainda foi possível determinar que o ducto apresentava $0,2 \mathrm{~cm}$ de abertura e $0,4 \mathrm{~cm}$ de ampola.

O tratamento de eleição para o PDA é através de intervenção cirúrgica (Johnson, 2007). De acordo com Fossum (2014) e Niebauer (1993) o animal deve ser posicionado em decúbito lateral direito e em seguida deve ser feito toracotomia lateral esquerda, no quarto ou quinto espaço intercostal, possibilitando acesso a região em que se encontra o ducto arterioso, deve ser posicionado o afastador e ser feito rebatimento dos lobos pulmonares para que após isso seja feita a incisão no saco pericárdico. O próximo passo é caracterizado pela identificação e isolamento do nervo frênico para evitar possíveis danos ao mesmo. Deve ser realizado dissecção do ducto até que o mesmo fique totalmente visível e em seguida é possível fazer a ligadura dupla com fio seda. As ligaduras devem der feitas com um intervalo de dois minutos para que a fisiologia se adeque ao novo volume sanguíneo e aumento da pressão arterial. De acordo com Bojrab (2005) após ligadura do ducto deve ser colocado o dreno torácico e prosseguir para rafia dos tecidos. A técnica escolhida para intervenção cirurgia no caso relatado teve como base a literatura descrita por se tratar de uma técnica muito utilizada e com grande probabilidade de êxito cirúrgico.

De acordo com Bonagura \& Lehmkukl (1999) e Belerenian et al. (2003) é possível constatar que $65 \%$ dos animais que apresentam PDA morrem antes de completarem um ano de vida caso não seja feita a correção cirúrgica, quando feito antes dos 6 meses o prognóstico é favorável. O êxito cirúrgico do caso do caso relatado, está relacionado a detecção precoce dessa cardiopatia congênita, realizada através do exame clínico durante a avaliação pediátrica completa, evidenciando sua importância para a resolução da anomalia e aumento na perspectiva de vida do animal. 


\section{Considerações finais}

No referente relato foi verificado a importância da avaliação clínica pediátrica para detectar a presença da anomalia congênita, favorecendo dessa forma o diagnóstico definitivo e realização do procedimento cirúrgico, levando consequentemente ao êxito cirúrgico e melhora clínica do animal estudado.

\section{Referências}

Belerenian, G. C., Mucha, C. J., \& Camacho, A. A. (2003). Afecções cardiovasculares em pequenos animais. Interbook.

Bojrab, M. J. (2005). Técnicas atuais em cirurgia de pequenos animais. Editora Roca.

Bonagura, J. D. (1992). Moléstia cardíaca congênita. In S. J. Ettinger (Ed.), Tratado de medicina interna veterinária: moléstias do cão e do gato (pp. 1026-1104). Manole Ltda.

Bonagura, J. D., \& Lehmkukl, L. B. (1999). Congenital heard diseases. In P. R. Fox, D. Sisson, \& N. S. Moise (Eds.), Textbook of canine and feline cardiology: Principles and clinical practice (pp. 471535). Sauders Company.

Broaddus, K., \& Tillson, M. (2010). Patent ductus arteriosus in dogs. Compendium Continuig Education for Veterinarians, 32(9), 1-14.

Buchanan, J. W., \& Patterson, D. F. (2003). Etiology of patent ductus arteriosus in dogs. Journal of Veterinary Internal Medicine, 17(2), 167-171. https://doi.org/10.1892/08916640(2003)017<0167:EOPDAI>2.3.CO;2.

Dehkordi, A. J., \& Hoseini, F. (2016). Patent ductus arteriosus in a lamb: A case report. Veterinary Research Forum, 7(1), 850-858.

Ecco, R., Snel-Oliveira, M. V, Barros, R. M., \& Santos, H. L. (2008). Patent ductus arteriosus in murrah buffalos. Veterinary Pathology, 45(4), 542-545.

Fossum, T. W. (2014). Cirurgia de pequenos animais (4th ed., Vol. 1). Elsevier Brasil.

Gournay, V. (2011). The ductus arteriosus: physiology, regulation, and functional and congenital anomalies. Archives of Cardiovascular Diseases, 104(11), 578-585.

Johnson, M. S. (2007). Options for treatment of patent ductus arteriosus in dogs. UK Vet Companion Animal, 12(1), 43-45. https://doi.org/10.1111/j.2044-3862.2007.tb00114.x.

Lee, B. H., \& Kim, W. G. (2004). A case of patent ductus arteriosus in a Holstein calf. Journal of Veterinary Science, 5(1), 83-84.

Niebauer, G. (1993). Técnicas atuais em cirurgia de pequenos animais. In M. J. Bojrab (Ed.), Current Techniques in Small Animal Surgery. Lea and Febiger.

Orton, C. e. (1997). Surgery of cardiovascular system. In Small animal surgery (pp. 575-608). Fossum, T W.

Saunders, J. H., Snaps, F. R., Peeters, D., Trotteur, G., \& Dondelinger, R. F. (1999). Use of a balloon occlusion catheter to facilitate transarterial coil embolisation of a patent ductus arteriosus in two dogs. Veterinary Record, 145(19), 544-546. https://doi.org/10.1136/vr.145.19.544 .

Snaps, F. R., Mc Entee, K., Saunders, J. H., \& Dondelinger, R. F. (1995). Treatment of patent ductus arteriosus by placement of intravascular coils in a pup. Journal of the American Veterinary Medical Association, 207(6), 724-725. https://doi.org/10.1111/j.1748-5827.1998.tb03630.x.

Strickland, K. N. (2008). Congenital heard diseases. In P. L. Tilley, F. W. K. Smith, M. A. Oyama, \& M. M. Sleeper (Eds.), Manual of canine and feline cardiology. Elsevier Health Sciences.

Histórico do artigo:

Recebido: 25 de janeiro de 2021

Aprovado: 16 de fevereiro de 2021
Licenciamento: Este artigoé publicado na modalidade Acesso Aberto sob a licença Creative Commons Atribuição 4.0 (CC-BY 4.0), a qual permite uso irrestrito, distribuição, reprodução em qualquer meio, desde que $\mathrm{o}$ autor e a fonte sejam devidamente creditados. 